

\title{
TUHOUSE: PROTOTIPO DE VIVIENDA SOCIAL SOSTENIBLE DE ALTA DENSIDAD PARA EL TRÓPICO
}

\author{
TUHOUSE: SUSTAINABLE, HIGH-DENSITY SOCIAL HOUSING \\ PROTOTYPE FOR THE TROPICS
}

\author{
CONSTANZA COBO FRAY \\ Magister en hábitat residencial \\ Docente de tiempo Completo \\ Universidad de San Buenaventura \\ Cali, Colombia \\ https://orcid.org/0000-0003-3883-4230 \\ ccobo@usbcali.edu.co
}

\author{
OLGA LUCIA MONTOYA FLÓREZ \\ Doctora en Arquitectura y Urbanismo \\ Docente de tiempo Completo \\ Universidad de San Buenaventura \\ Cali, Colombia \\ https://orcid.org/0000-0003-2814-0382 \\ olmontoy@usbcali.edu.co
}




\section{INTRODUCCIÓN}

El prototipo TUHOUSE es producto de una reflexión académica sobre el papel que cumple la vivienda social en la constitución del hábitat residencial y en su compromiso con el mejoramiento del medio ambiente y de diferentes ámbitos de la calidad de vida. TUHOUSE propone un modelo de vivienda social replicable, flexible y adaptable a diferentes contextos y a diferentes poblaciones. El concepto de sostenibilidad y las estrategias bioclimáticas se relacionan directamente con las decisiones urbanoarquitectónicas, en la medida en que la propuesta incorpora criterios de sostenibilidad, implementando agricultura urbana y sistemas de compostaje en los espacios comunes, favoreciendo así la conformación de comunidad y contribuyendo a generar seguridad alimenticia e ingresos adicionales para las familias.

Las edificaciones se proponen en un sistema estructural y constructivo de grandes piezas de prefabricados en Concreto con Agregados Reciclados (CAR), lo que busca sustituir la explotación de materias primas no renovables y disminuir el impacto de los Residuos de Construcción y Demolición en el paisaje (Bedoya y Dzul, 2015). En Colombia se usan una variedad de materiales para la construcción de vivienda social en serie, de los cuales el 99\% se basa en mampostería de alta densidad como el concreto (Giraldo, Czajkowski y Gómez, 2020).

Entendiendo la vivienda social como el bien más preciado de los habitantes, esta debe solucionar las necesidades de índole sociocultural: dar cobijo (considerando distintos modos de vida y costumbres), poder transformarse para acoger los diferentes tipos de familias y su crecimiento, y ser durable (la vivienda es para toda la vida, es la herencia de la familia). Pero también debe contemplar necesidades de índole económica: valorización de la vivienda, rentabilidad y generación de ingresos extras, entre otras.

Además de los compromisos propios de la disciplina, hoy en día la Arquitectura también tiene compromisos con el cuidado del medio ambiente, la conservación del planeta, la eficiencia energética y el confort. Sin embargo, algunas viviendas de interés social en Cali exhiben un paisajismo pobre y carecen de estrategias bioclimáticas, alcanzando temperaturas hasta de $49^{\circ} \mathrm{C}$ en su interior (Gamboa, Rosillo, Herrera, López y Iglesias, 2011) y, desde luego, un alto disconfort para sus habitantes. Como bien señala Montoya (2014), en general los proyectos presentan baja exploración tipológica y condiciones deficientes en cuanto a su orientación solar, a sus elementos de protección en los espacios comunes, así como a sus fachadas y cubiertas. Desafortunadamente, en la mayoría de proyectos actuales, se evidencia una baja implementación de estrategias bioclimáticas y sostenibles, como correcta orientación, sombreo en fachada, ventilación natural, las cuales quedan reservadas para un menor número de viviendas correspondientes a otros sectores económicos más favorecidos de la población.

Además, el sector residencial consume alrededor del $20 \%$ del total de la energía en el país (Unidad de Planeación Minero Energética [UPME], 2019) y de ese consumo -dependiendo de la condición económica-, entre el $40 \%$ y el $60 \%$ se destina a climatizar el ambiente a través del aire acondicionado, la refrigeración y el uso de ventiladores (UPME, 2018).

El prototipo TUHOUSE fue construido por estudiantes y docentes, en escala 1:1, en el marco del concurso Solar Decathlon para América Latina y el Caribe (LAC) 2019, y recoge el aprendizaje realizado por el equipo en la versión anterior (año 2015) con el prototipo MIHOUSE. En dicho prototipo se inicia la exploración arquitectónica y bioclimática del concreto como material de la envolvente (Cobo, Villalobos y Montoya, 2019), y se dan los primeros ensayos en el aprovechamiento y reutilización de las aguas, el manejo de residuos a través de compostera casera y la incorporación de la energía solar a través de paneles solares dispuestos en la cubierta (López y Holguín, 2020). Lo anterior permite presentar al público en general (no científico) alternativas posibles de ser incorporadas, extendiendo el rol de la academia por fuera de los límites de la Universidad. En ese sentido, cabe agregar que el proyecto generó alianzas no solo entre universidades, sino con el sector público y el empresarial.

Es pertinente entonces en esta revisión, remitirse al modelo de confort térmico denominado adaptivo y propuesto por la norma internacional ASHRAE en su última versión (ASHRAE/ANSI, 2017) para edificios ventilados naturalmente. Este modelo liderado por autores como Auliciems (1975) y Nicol, Humphreys y Roaf (2012) y que se basa en el promedio de temperatura ambiente de un lugar, surge como crítica a los rangos establecidos en condiciones controladas, propios del modelo analítico promovido por ASHRAE (ASRHAE, 2005) y retomados para Colombia en la NTC 5316 (Instituto Colombiano de Normas Técnicas y certificación -ICONTEC, 2004). El modelo analítico resulta de investigaciones realizadas en laboratorio en contextos con las cuatro estaciones climáticas (Fanger, 1972; Fanger y Toftum, 2002), por lo que es necesaria su revisión en otros contextos, como los tropicales (Herrera y Rosillo, 2019), tal y como lo evidencian estudios recientes en colegios en el trópico (Zapata et al., 2018)

A continuación, se presentan los aspectos sostenibles y bioclimáticos de la propuesta, como también un análisis de los parámetros exigidos por el concurso (temperatura entre $22^{\circ} \mathrm{C}$ a $25^{\circ} \mathrm{C}$ y humedad relativa entre $40 \%$ a $60 \%$ ) para alcanzar el confort térmico, los cuales poco corresponden con la habitabilidad de los espacios ventilados naturalmente durante todo el año. 


\begin{tabular}{|c|c|}
\hline Parámetro & Valores establecidos \\
\hline Temperatura ambiente interior $(\mathrm{Ta})$ & $22^{\circ} \mathrm{C}$ a $25^{\circ} \mathrm{C}$ \\
\hline Humedad relativa (\%) & $40 \%$ a $60 \%$ \\
\hline
\end{tabular}

Tabla 1. Parámetros térmicos propuestos por el Concurso Solar Decathlon LAC 2019. Fuente: Solar Decathlon (2019).

\section{METODOLOGÍA}

La propuesta urbano-arquitectónica en cuestión surge en el taller de proyectos de cuarto año del Programa de Arquitectura, la cual toma como marco referencial el concurso internacional Solar Decathlon LAC 2019, entidad que promueve la investigación universitaria en temas de vivienda social sostenible, poniendo énfasis en utilización de energías renovables, confort y protección del medio ambiente. El proyecto realizado para el concurso se llamó TUHOUSE y para su desarrollo, de dos años y medio, se conformó un equipo multidisciplinar de estudiantes (50) y profesores (10) pertenecientes a dos universidades de la región (Universidad San Buenaventura-Cali y Universidad Autónoma de Occidente) con conocimientos complementarios en áreas de arquitectura bioclimática, hábitat, agricultura urbana, medio ambiente, sostenibilidad, energías renovables. Ello permitió no solo abarcar todos los temas solicitados por el concurso, sino abordarlos de manera innovadora a través de una mirada interdisciplinaria (Baumber, Kligyte, Bijl-Brouwer, Van Der y Pratt, 2020; Herrera, Rey, Hernández y Roa, 2020).

Las etapas principales de trabajo fueron: a) fundamentos urbanos y arquitectónicos acordes con el lugar y la población; b) simulación térmico-energética; c) costos; d) prefabricación y construcción de un prototipo de vivienda a escala $1: 1 ; y$, e) monitoreo y verificación del funcionamiento de las estrategias.

Metodológicamente, para la fase de diseño, la propuesta se desarrolló en un taller-laboratorio de investigación aplicada que integra lo proyectual, lo sostenible y lo bioclimático, alternando los procesos de diseño con la verificación a partir de simulaciones en softwares (como Formit y Design Builder) y observaciones en el laboratorio de bioclimática (heliodón y mesa de humo). Una vez que el prototipo fue construido en una fase posterior, se realizaron una serie de mediciones en sitio. Esta metodología, propia del proceso bioclimático (San Juan et al., 2013) que incorpora análisis bioclimático, dimensionamiento inicial y final y mediciones para contrastar hipótesis, resulta ser muy enriquecedora en el proceso de aprendizaje de los estudiantes, ya que pasa del diseño convencional al diseño energéticamente optimizado (Montoya, 2020) y trasciende el aprendizaje en el aula al originar la posibilidad de confrontar los conocimientos recibidos con el hecho real y de verificar los resultados.
Después de que se elaboró el prototipo en el lugar determinado por el concurso, denominado Villa Solar, se realizaron mediciones interiores continuas de temperatura ambiente $\left({ }^{\circ} \mathrm{C}\right)$, humedad relativa (\%), calidad de aire (CO2), iluminancia (lux) y generación de energía con equipos especializados dispuestos por el mismo concurso. Además, se hicieron mediciones puntuales sobre elementos característicos de la envolvente, al exterior y al interior, con termómetro infrarrojo marca Nubee. El consumo de energía (kWh) se midió a través de tareas específicas en el prototipo, que implicaran el uso de aparatos y electrodomésticos. Los parámetros acústicos, como el nivel de presión sonora (dB) y el tiempo de reverberación, se midieron en un día específico por parte de los organizadores con equipos especializados. En cuanto a confort térmico se refiere, se requería alcanzar los valores indicados en la Tabla 1.

Para el análisis de confort térmico, se comparó el rango propuesto por la competencia con el rango propuesto por el modelo adaptativo (ASHRAE/ANSI, 2017), indicado en la Ecuación 1, así:

Tacep $=$ $0.31 * \mathrm{~T}(\mathrm{pma}$ (out) $) \pm 17.8 \pm \mathrm{T} \_\lim (1)$

Donde:

Tacep $=$ Temperatura aceptable

$T($ pma(out) $=$ Promedio de temperatura exterior T_lim = Límites de Temperatura, los cuales pueden ser de \pm 3.5 para una aceptabilidad del 90\%. (Nicol et al., 2012).

\section{RESULTADOS Y DISCUSIÓN}

\section{LA PROPUESTA URBANA}

La propuesta urbana está basada en un diseño sustentable de un conjunto de viviendas sociales en altura, de 5 y 8 pisos, y una densidad de 120 viviendas/hectárea. El conjunto se desarrolla a partir de un sistema urbano de agrupaciones de edificios de viviendas que conforman espacios urbanos públicos y privados, capaz de adaptarse a distintos contextos sociales y climáticos propios de cada lugar. En algunos casos, el espacio común por excelencia es el recinto y en otros, la calle; ambos propicios para fortalecer el encuentro, la identidad y la convivencia. En palabras de Samper: "Lo fundamental no son los diseños propiamente dichos de las viviendas, sino la búsqueda de nuevos patrones urbanos. Trabajar en la búsqueda de nuevos patrones urbanos lleva implícito encontrar nuevas tipologías de viviendas" (2003, p. 20). 


\begin{tabular}{|c|c|c|}
\hline Superficie & Expuesta al sol & Bajo sombra \\
\hline Concreto & $49.2^{\circ} \mathrm{C}$ & $28.3^{\circ} \mathrm{C}$ \\
\hline Piso adoquín exterior & $39.4^{\circ} \mathrm{C}$ & $29.1^{\circ} \mathrm{C}$ \\
\hline Tierra con vegetación & $35.4^{\circ} \mathrm{C}$ & $27.0^{\circ} \mathrm{C}$ \\
\hline
\end{tabular}

Tabla 2. Reducción en temperaturas proyecto TUHOUSE. Fuente: Elaborado por los autores.

La disposición cuidadosa de los edificios (orientación, distanciamiento y altura) logra conformar espacios sombreados que, acompañados de vegetación y arborización nativa, permiten la disminución significativa de la incidencia solar, generando un microclima adecuado. Los vientos juegan un papel crucial a la hora de disipar el calor producido por los materiales y por los mismos elementos del proyecto. La sucesión de espacios amplios (recintos) y espacios estrechos (calles y accesos) produce el llamado "efecto Venturi" que, junto a la presencia de fachadas verdes en los lugares estrechos, logra un enfriamiento pasivo de los vientos que ingresan a las agrupaciones. Todas son estrategias para el clima cálido de la ciudad de Cali recomendadas por autores emblemáticos (Olgyay, 1963) (Figura 1). Según las mediciones tomadas in situ, estas estrategias logran disminuir las temperaturas hasta en 10 grados (Tabla 2).

El conjunto de vivienda cuenta, además, con un equipamiento cultural, educativo y productivo; un sistema de movilidad en bicicleta conectado a la red de ciclo-rutas de la ciudad; y un sistema de huertas urbanas productivas y árboles frutales, que generan ingresos adicionales a los habitantes del conjunto y propician calidad ambiental. En el entendido de que la calidad de vida no se limita exclusivamente a la habitabilidad al interior de la vivienda, se busca minimizar posibles impactos negativos y promover el uso sostenible de espacios comunes (Cobo et al., 2019) (Figura 2).

\section{LA PROPUESTA ARQUITECTÓNICA Y SOSTENIBLE}

Esta propuesta considera que la vivienda social debe cumplir con 4 condiciones básicas para ser habitable y sustentable: la vivienda debe ser progresiva y productiva (Samper, 2002), además de replicable y flexible. Principios fundamentales que permiten el libre desempeño de los modos de habitar de las diferentes familias. Los espacios de la vivienda deben posibilitar adecuarse a las necesidades cambiantes de la familia, tal y como lo propone el equipo AURA (Universidad de Sevilla, España y Universidad Santiago de Cali, Colombia), el cual implementa sistemas modulares progresivos y espacios flexibles determinados por el mobiliario (Herrera, Pineda, Roa, Cordero y López, 2017). Y no como ocurre con las ofertas actuales en donde las familias se deben adecuar a la vivienda. A fin de alcanzar esta cualidad, las viviendas deben ser

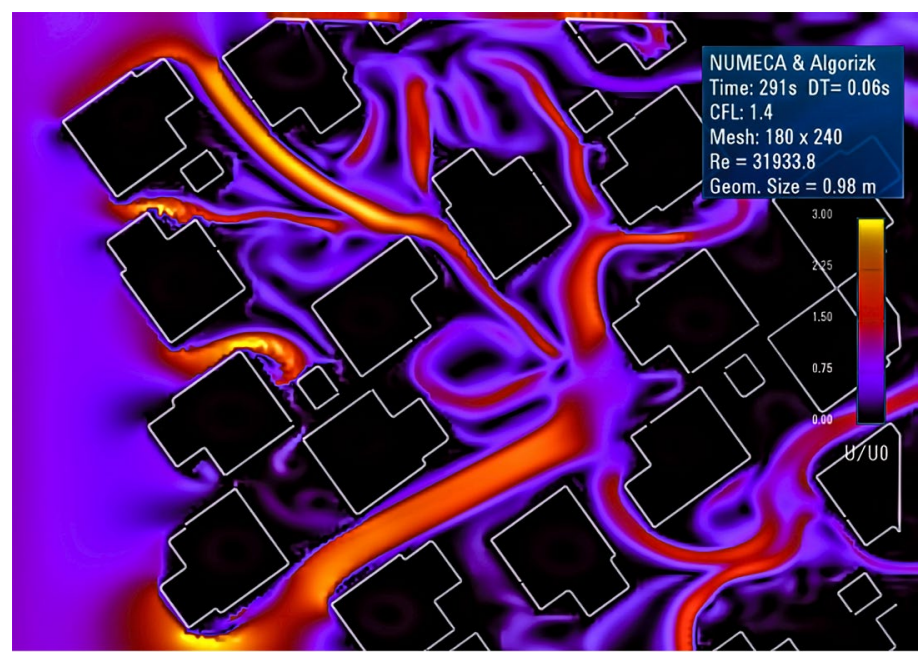

Figura 1. Comportamiento de los vientos. Wind Tunnel. Fuente: Elaborado por los autores.

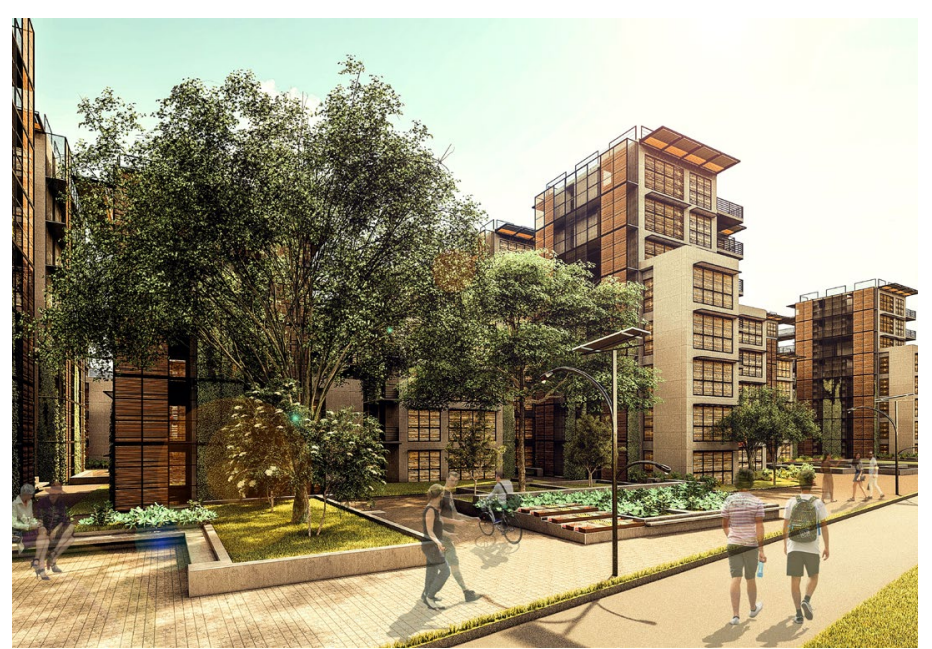

Figura 2. Imagen del espacio público del conjunto. Fuente: Elaborado por los autores

planteadas desde el principio para ser susceptibles a las ampliaciones y remodelaciones que la familia considere necesarias y para que, incluso, puedan convertirse en una fuente de ingresos, en viviendas productivas. Se trata de principios que se pusieron a prueba desde la pasada versión del concurso Solar Decathlon, en el año 2015, y que demostraron su factibilidad urbana y arquitectónica como sistema (Cobo et al., 2019). 


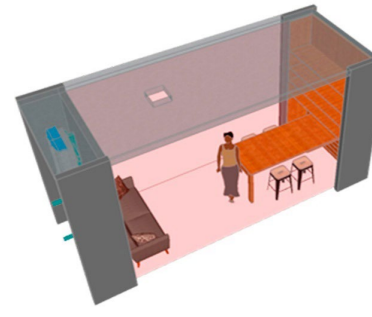

Módulo 1 Habitación
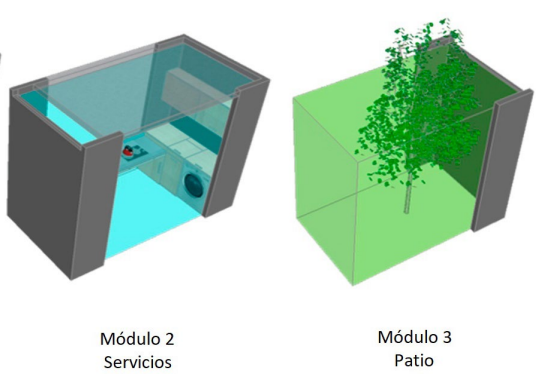

Figura 3. Módulos del prototipo. Fuente: Elaborado por los autores.
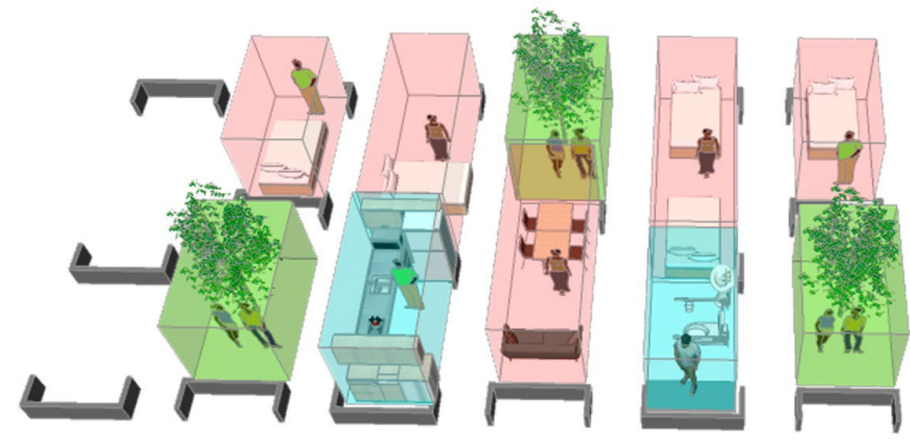

Figura 4. Flexibilidad y progresividad. Fuente: Elaborado por los autores.

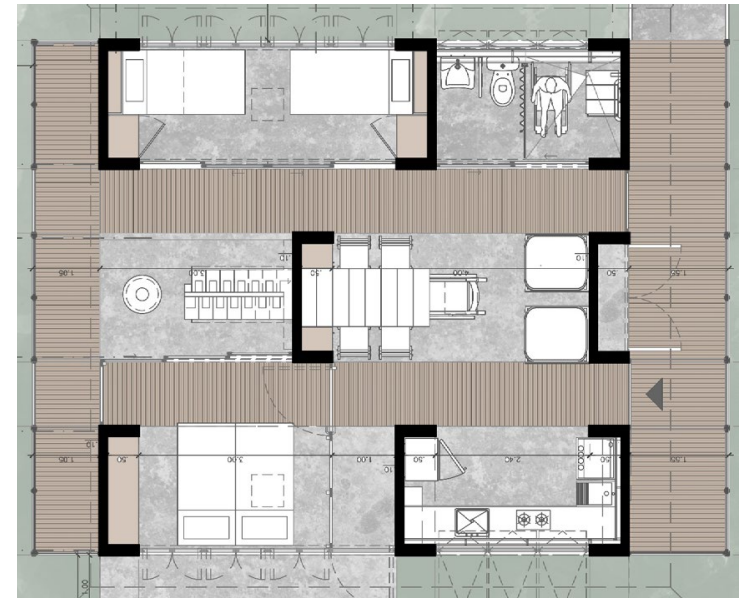

Figura 5. Planta. Fuente: Elaborado por los autores.

El proceso proyectual también debe ser sustentable: optimizar procesos de diseño para luego optimizar procesos constructivos y de utilización de recursos. Es por esto que la unidad de vivienda propuesta se concibe como un "lego", conformado por dos módulos base, en los cuales se define el espacio-forma-estructura en un mismo elemento (Figura 3).

En el módulo de mayor tamaño se disponen las habitaciones (dormitorio, comedor, salón, estudio) y en el módulo pequeño, los servicios (cocina, baño, patio). La manera en que estas unidades se ordenan en relación a un sistema de circulación y alrededor de un patio permite conformar variedad de unidades de vivienda, flexibles y progresivas, tanto en su uso como en su construcción, para acoger a diferentes tipos de familias (Figura 4). Esta disposición permite igualmente aprovechar las condiciones ambientales que proporcionan los patios de las casas tradicionales de la región, como sombra, ventilación, humedad y enfriamiento por evaporación (Figura 5), de manera similar a otros prototipos del Solar, como Patio 2.12, el cual retoma la casa tradicional andaluza e incorpora estrategias pasivas que respondan, entre otros factores, al verano caluroso, de forma similar al caso presentado (Terrados, Baco y Moreno, 2015).
Figura 6. Corte. Fuente: Elaborado por los autores.

Junto con proporcionar cobijo, la vivienda planteada genera alimentos (en su techo y fachadas), confort y energía. La propuesta reutiliza aguas grises y recolecta aguas lluvias; utiliza materiales y tecnología adecuados para el sostenimiento del medio ambiente; es coherente y eficaz en diferentes contextos y afronta los problemas de densidad urbana (Figura 6).

Adicionalmente, se trata de un proyecto autosustentable y eficiente, donde cada elemento que lo compone cumple varias funciones. Las fachadas este-oeste y la cubierta (las más afectadas por la incidencia del sol) se recubren por una envolvente verde que cumple tres funciones principales: aislar la estructura de concreto de la incidencia solar-que constituye una de las principales recomendaciones para el clima tropical (Evans y Schiller, 1994; Konya, 1980)-, producir alimentos y purificar el aire, contribuyendo a la disminución de la contaminación del medio ambiente. El diseño incluye un espacio de transición bioclimático entre el interior y el exterior (Figuras 7 y 8 y Tabla 3), que se asemeja al espacio del zaguán de las casas tradicionales de la región (Herrera et al., 2017). 


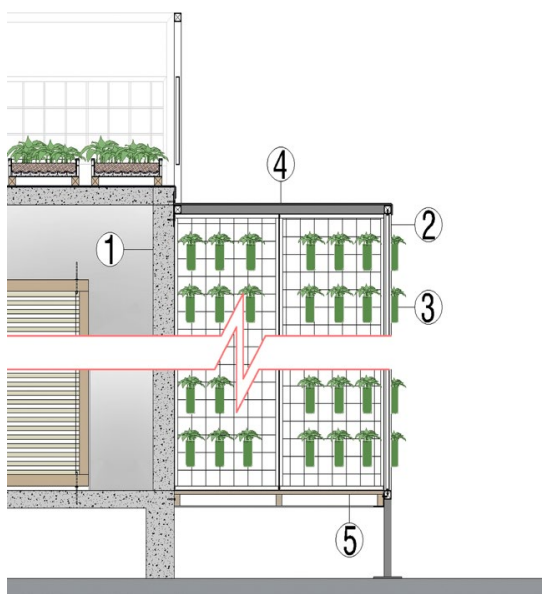

Figura 7. Corte por fachada. Fuente: Elaborado por los autores.

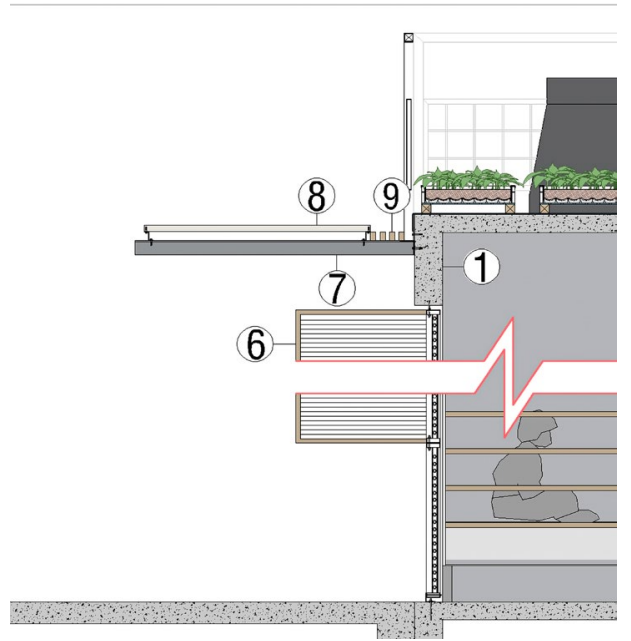

Figura 9. Corte por fachada. Fuente: Elaborado por los autores.

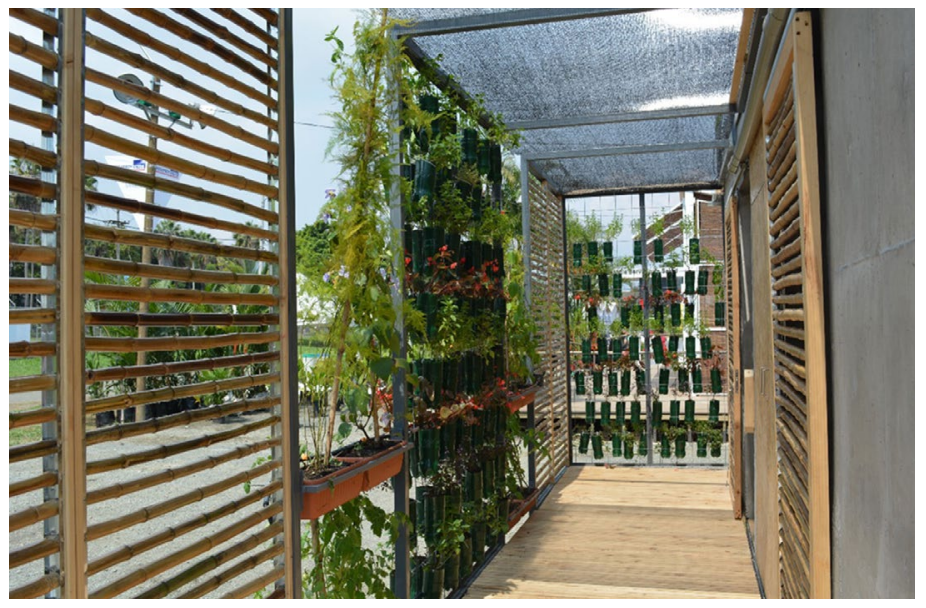

Figura 8. Espacio de acceso, envolvente verde. Fuente: Elaborado por los autores.

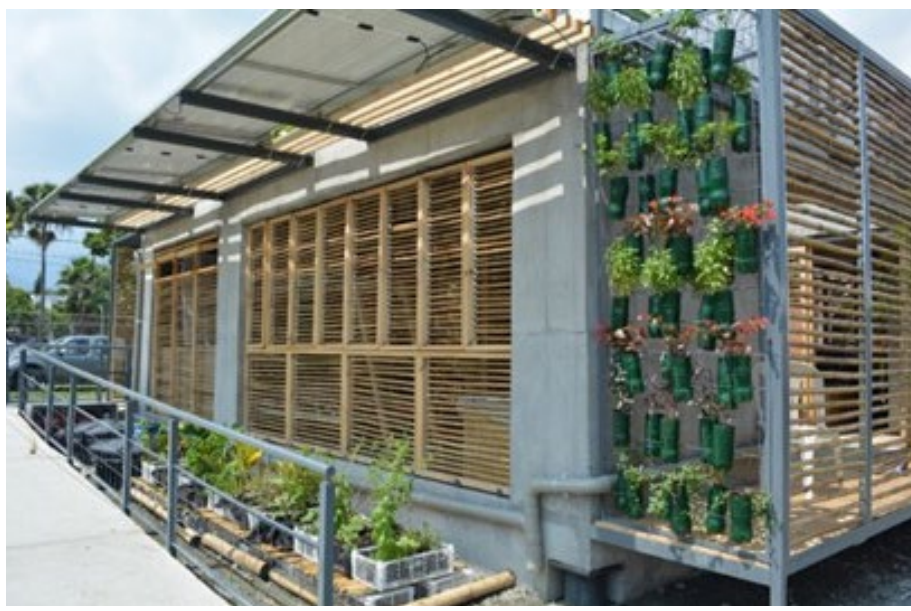

Figura 10. Fachada sur. Fuente: Elaborado por los autores.

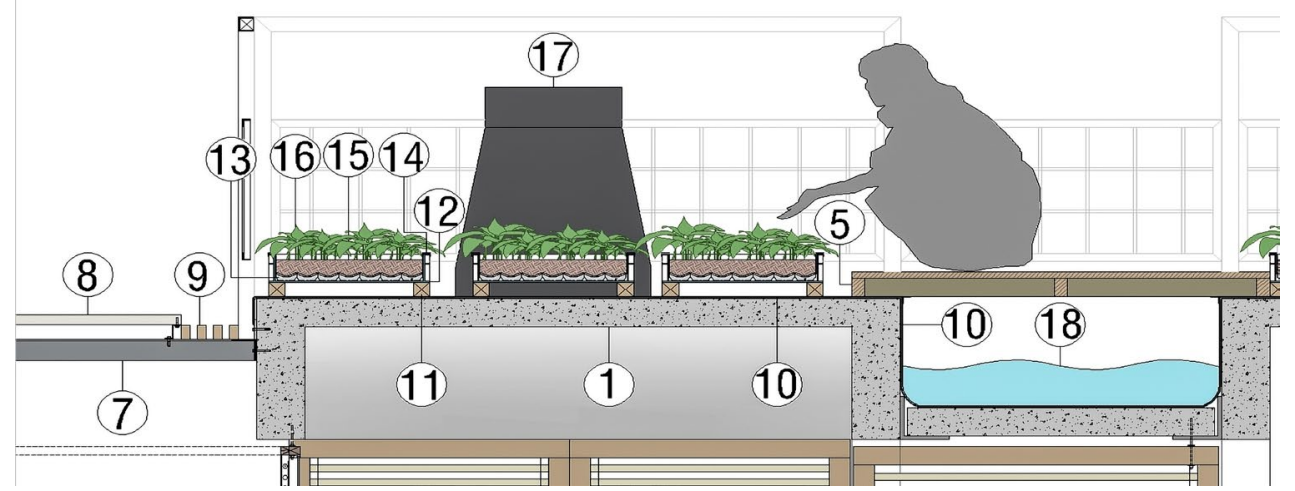

Figura 11. Detalle de cubierta de vivienda propuesta. Fuente: Elaborado por los autores. 


\begin{tabular}{|c|c|c|c|c|}
\hline Ubicación & No. & Materiales especificación & $\begin{array}{l}\text { Espesor } \\
(\mathrm{mm})\end{array}$ & $\begin{array}{l}\text { Conductividad } \\
\text { térmica }(\mathrm{W} / \mathrm{m} . \mathrm{k})\end{array}$ \\
\hline Muro & 1 & Concreto reforzado 3000 Psi & 10 & 0.97 \\
\hline \multirow{2}{*}{ Jaula fachada } & \multirow[t]{2}{*}{2} & Malla metálica electro-soldada & 5 & 58 \\
\hline & & Bambú & 60 & 0.28 \\
\hline \multirow[t]{3}{*}{ Fachada verde } & \multirow[t]{3}{*}{3} & Botellas plásticas PET recicladas & 2 & $0.24^{\star \star}$ \\
\hline & & Tierra & 60 & $0.8^{*}$ \\
\hline & & Plantas aromáticas & - & - \\
\hline Ubicación & No. & Materiales especificación & $\begin{array}{l}\text { Espesor } \\
(\mathrm{mm})\end{array}$ & $\begin{array}{l}\text { Conductividad } \\
\text { térmica }(\mathrm{W} / \mathrm{m} \cdot \mathrm{k})\end{array}$ \\
\hline Cubierta de Jaula & 4 & Polisombra negra 35\% (LXA) Anti-UV (Polietileno) & 1 & 0.35 \\
\hline Piso en estibas & 5 & Madera & 25.5 & 0.15 \\
\hline \multirow[t]{2}{*}{ Ventanas Fachada } & \multirow[t]{2}{*}{6} & Listón madera & 25.5 & 0.15 \\
\hline & & Palos de bambú & 60 & 0.28 \\
\hline \multirow{3}{*}{ Alero } & 7 & Estructura metálica soporte de paneles solares & 38.1 & 0.28 \\
\hline & 8 & Panel solar & 70 & 1.05 \\
\hline & 9 & Cuartones de madera en pino radiata & 50.8 & 0.28 \\
\hline \multirow[t]{9}{*}{ Cubierta } & 10 & $\begin{array}{c}\text { Membrana impermeabilizante TPO SINTOFOIL SL (EELAB } \\
\text { certificado) (etileno-propileno) }\end{array}$ & 1.2 & 0.24 \\
\hline & 11 & Escalerillas en madera pino radiata & 50.8 & 0.28 \\
\hline & 12 & Canastas plásticas recicladas & 25.4 & 0.50 \\
\hline & 13 & Base de botellas PET personales & 2 & 0.24 \\
\hline & 14 & Geotextil NT 1600S (polipropileno) & 1.5 & 0.24 \\
\hline & 15 & Tierra húmeda & 150 & $0.8^{\star}$ \\
\hline & 16 & Plantas & - & - \\
\hline & 17 & Chimenea solar en lámina metálica (aluminio) & 5 & 204 \\
\hline & 18 & Canal de agua en concreto & 10 & 0.97 \\
\hline
\end{tabular}

Tabla 3. Conductividad térmica de los materiales utilizados en la construcción del prototipo TUHOUSE. Fuente: IRAM 11601 (2002); Van der Vegt y Govaert (2005).

Las fachadas sur-norte están abiertas para aprovechar la circulación cruzada de los vientos -otra estrategia sugerida para el clima tropical (Olgyay, 1963)- y están protegidas por grandes aleros que, además de producir sombra y proteger de la lluvia, soportan los paneles solares que proveen energía solar a la vivienda (Figuras 9 y 10 y Tabla 3).

A la quinta fachada se le debe prestar especial atención, sobre todo en el contexto de Cali, por sus condiciones climáticas cálidas, pero también por las condiciones sociales (Sánchez, 2019). Se diseñó aquí una huerta en cubierta que es aprovechada para recolectar aguas lluvias en dos grandes canales ubicados bajo las estibas de madera removibles y que sirven para la circulación (Figura 11). Esta técnica permite, asimismo, airear la cubierta a través de una cámara ventilada (Tabla 3), como estrategia bioclimática enfocada en la superficie horizontal, la cual recibe al menos el $50 \%$ de la radiación solar en latitudes tropicales (Olgyay, 1963).

A partir de los criterios anteriores, se refuerza la hipótesis sostenida por Becker, Goldberger, y Paciuk (2007), quienes plantean que los aspectos de diseño con mayor impacto en la climatización son la orientación y las áreas de aberturas en las fachadas, y la resistencia térmica de los muros y del techo.

\section{MEDICIONES PARÁMETROS AMBIENTALES $Y$ ENERGÉTICOS}

Las mediciones de temperatura ambiente ( $\mathrm{Ta}$ ) al exterior arrojan valores de $24.5^{\circ} \mathrm{C}$ promedio, con máximas que alcanzan $32.4^{\circ} \mathrm{C}$ y mínimas de $18.2^{\circ} \mathrm{C}$. Mientras que la humedad relativa exterior promedio resulta de $74.5 \%$, con valores máximos de $94 \%$ y mínimos de $45 \%$. Estos valores resultan consistentes con las condiciones climáticas locales, 


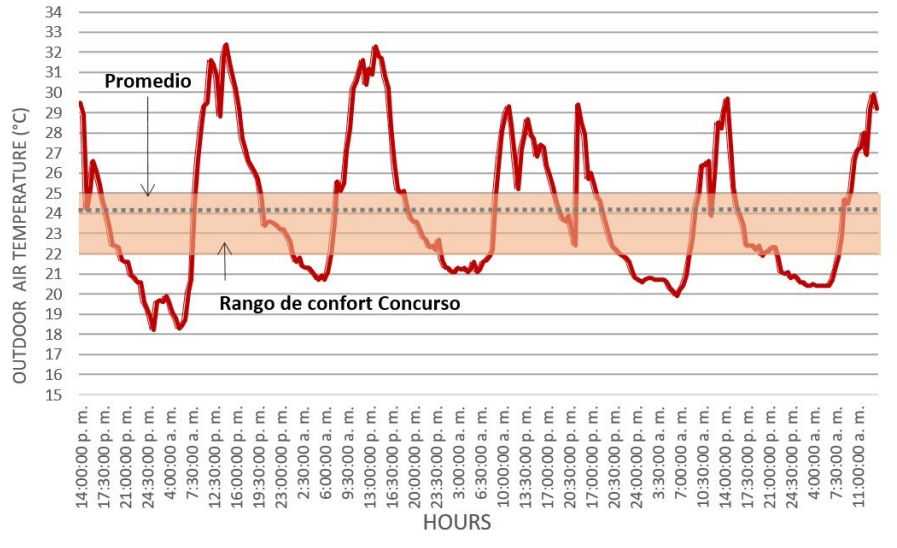

Figura 12. Temperatura exterior. Fuente: Elaborado por los autores.

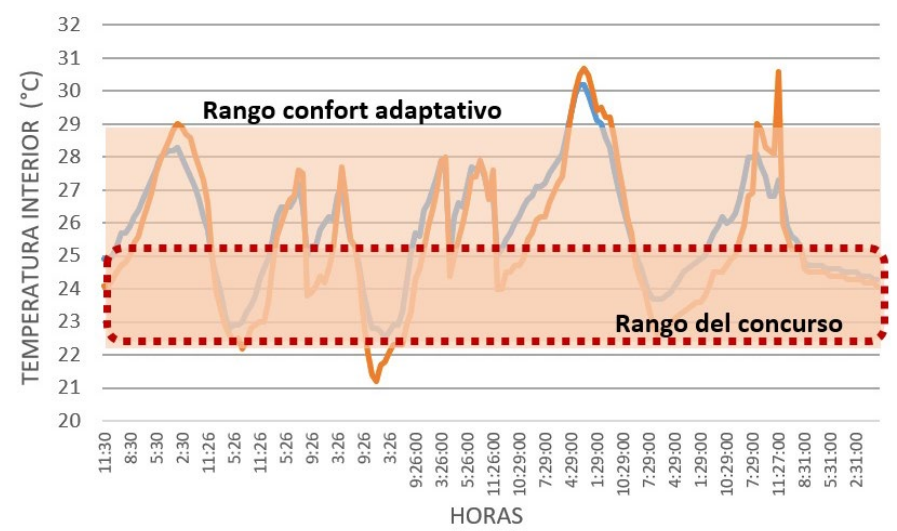

Figura 14. Temperatura al interior del prototipo. Fuente: Elaborado por los autores.

las cuales presentan mínimas variaciones durante el año, propias de contextos tropicales y ecuatoriales como el de este estudio.

En las Figuras 12 y 13 se presentan los registros tomados durante los 7 días de competencia. En estas mismas gráficas es posible observar los valores de confort higro-térmico indicados por el concurso, y lo alejados que pueden resultar de las condiciones locales, propias de regiones tropicales.

En cuanto al comportamiento de los espacios al interior del prototipo, se puede observar en la Figura 14 que, a pesar de conservar una temperatura interior promedio de $25^{\circ} \mathrm{C}$, son solo las horas de la mañana y la madrugada las que logran estar en el rango de confort propuesto por el concurso. Por otro lado, si el análisis de confort se realiza a partir del rango propuesto por el modelo adaptivo, el cual resulta entre $21.8^{\circ} \mathrm{C}$ y $28.8^{\circ} \mathrm{C}$ (véase Ecuación 1), propio de contextos tropicales y de edificios con ventilación natural, vemos que la mayoría de los días y las temperaturas quedan dentro del rango.

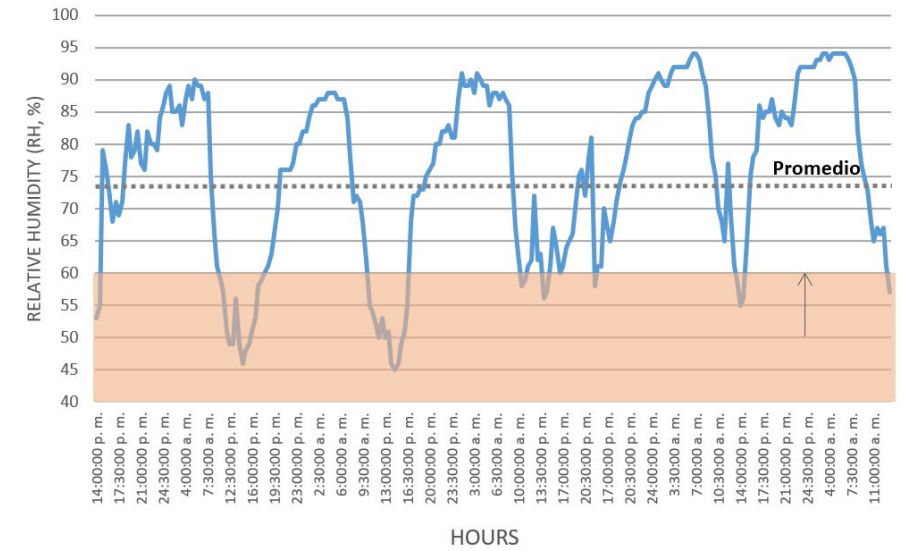

Figura 13. Humedad relativa exterior. Fuente: Elaborado por los autores.

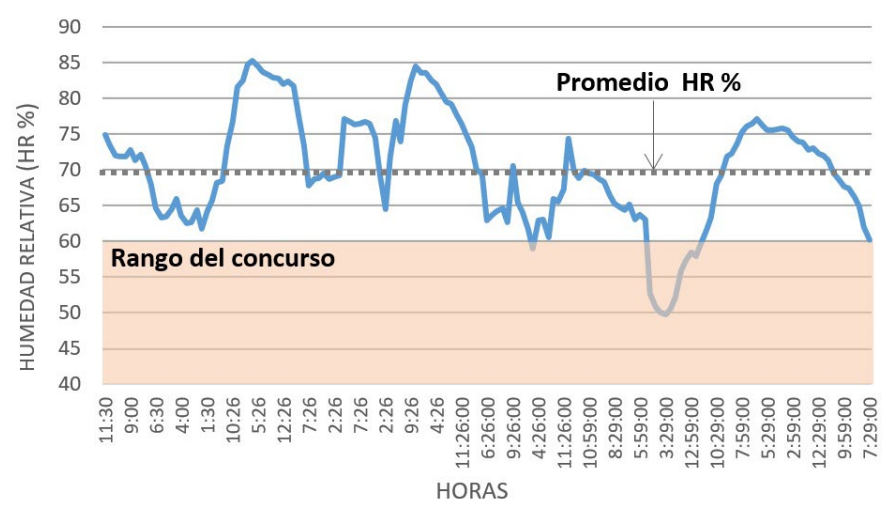

Figura 15. Humedad relativa al interior del prototipo. Fuente: Elaborado por los autores.

En cuanto a la humedad relativa, los valores estuvieron alejados del rango del concurso y cercanos a los registrados al exterior, lo cual corresponde a edificios ventilados naturalmente. Como se presenta en la Figura 15, el promedio de $70 \% \mathrm{HR}$ al interior del prototipo estuvo cercano al promedio exterior registrado durante los mismos días (75 \%HR).

Enfocando el análisis de las mediciones durante un día típico de la competencia, es posible observar (Figura 16) que, según el rango propuesto por el concurso, el prototipo se encuentra en confort solo en la noche y al inicio de la mañana. Mientras que, bajo el modelo adaptivo, solo entre las horas de la tarde (1:30 a 4:30), con temperaturas cercanas a $29^{\circ} \mathrm{C}$, este se escapa del rango confort. Lo anterior tiene implicaciones energéticas importantes, dado que frente a una mayor exigencia de confort -como es el caso del análisis de acuerdo al rango indicado por el concurso- retomada de la norma internacional (diseñada para otros contextos con estaciones climáticas marcadas), se puede asumir un diseño térmico que implique un mayor consumo energético derivado de la necesidad de refrigerar las condiciones internas de la vivienda. 


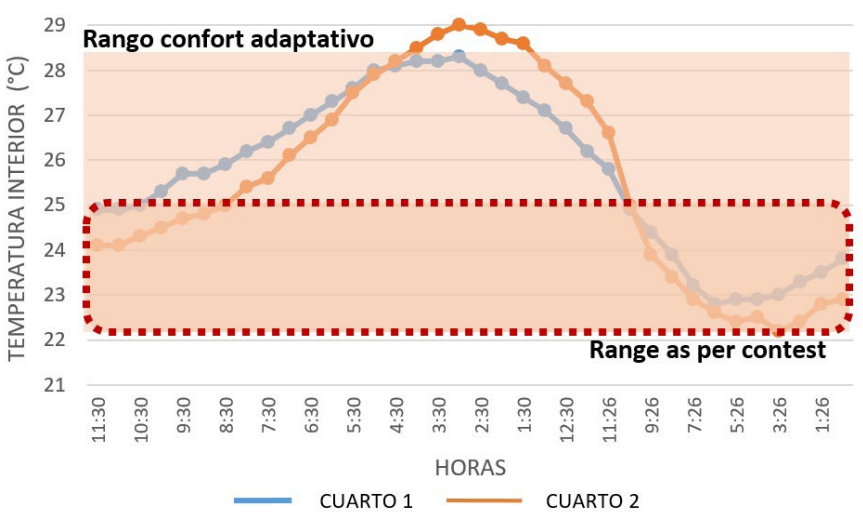

Figura 16. Temperatura día típico al interior del prototipo TUHOUSE. Fuente: Elaborado por los autores.

De igual forma, se implementan paneles fotovoltaicos que captan energía solar para transformarla en energía eléctrica, generando viviendas autosuficientes en el consumo eléctrico, con el fin de reducir las emisiones de dióxido de carbono por medio del sistema fotovoltaico que produce anualmente $3578 \mathrm{kWh}$. Mediante el uso eficiente de la energía eléctrica se reduce el consumo en el proyecto casi un 40\%, que es igual a $1166 \mathrm{kWh}$ ahorrados en el año.

El consumo energético diario promedio del prototipo se mantuvo durante los ocho días de la competencia en $58 \mathrm{kWh}$, respondiendo al objetivo del concurso de mantenerlo por debajo de $70 \mathrm{kWh}$. Esto se logró gracias a electrodomésticos de alta eficiencia y a bombillas LED para la iluminación nocturna. En cuanto al balance energético, ideal en valor $0 \mathrm{kWh}$, resultó aquí en $-3.4 \mathrm{kWh}$, como diferencia entre la energía exportada a la red (35.98 $\mathrm{kWh}$ ) y la importada (39.39 kWh), lo que permitió alcanzar el tercer lugar en esta prueba (Macías, 2020).

En términos de sustentabilidad, se plantea el re-uso de aguas grises (previo tratamiento) y el aprovechamiento de aguas lluvias para actividades que no requieran agua potable y que representan un elevado porcentaje en el consumo diario del hogar, tales como el uso de sanitarios y el riego de zonas verdes y jardines. Las aguas grises de la ducha y del lavamanos se recogen, en un dispositivo llamado "ecoguardián" para reusarlas en labores de aseo. También se implementa un sistema de recolección interceptor y tanque de almacenamiento de aguas Iluvias. Todo esto con el propósito de disminuir el consumo de agua potable de un $16 \%$ a un $40 \%$ y la cantidad de vertimiento al alcantarillado. Las anteriores estrategias buscan aminorar los costos del servicio de agua potable y alcantarillado.

El proyecto reduce la generación de residuos hasta su disposición final, realizando una correcta separación de los residuos en la fuente y disponiendo los residuos orgánicos en una unidad de compostaje. A partir de esas dos medidas, se aprovechó alrededor del $80 \%$ de lo generado. Paralelamente, se proponen estrategias para el manejo adecuado de los residuos sólidos generados en cada una de las fases: construcción, operación y demolición.

Como un aporte a la sostenibilidad, el prototipo de vivienda se construye con un sistema de grandes piezas prefabricadas de concreto elaborado con diversos componentes considerados residuos industriales, como cenizas de alto horno o provenientes de la quema del bagazo de caña de la industria de la región, insumos que permiten además la mejora de resistencias en las mezclas utilizadas. Una vez cumplida su vida útil, este concreto no sólo puede convertirse en material de reciclaje para la fabricación de elementos no estructurales, como adoquines para pisos, eventualmente las piezas completas pueden ser reutilizadas en otro tipo de construcción, lográndose, desde este punto de vista, una alta sostenibilidad.

\section{CONCLUSIÓN}

El proceso de diseño bioclimático implementado en el curso transversal e interinstitucional permitió poner a prueba las decisiones de diseño tomadas a través de mediciones y simulaciones, para finalmente aplicar el conocimiento adquirido en la construcción del prototipo en la Villa Solar. Lo anterior fue posible gracias a concursos como el Solar Decathlon, el cual fomenta una dinámica de aprendizaje teórico-práctico, imprescindible para las Facultades de Arquitectura.

En este marco, se verificó el comportamiento exitoso de estrategias arquitectónicas al momento de proporcionar confort: sombreo de las superficies en concreto expuestas a la radiación, constitución de masa térmica especialmente en la cubierta a través de la huerta, envolvente verde, ventilación cruzada, fachada permeable para la ventilación y renovación constante de aire. Estrategias que, ciertamente, fueron exploradas para otros prototipos del concurso a través de interesantes variaciones y aplicaciones.

A través del prototipo expuesto se demostró el buen desempeño térmico del concreto; evidencia relevante si se tiene en cuenta que es el principal material con el que se construye la vivienda social en la actualidad -a través de estrategias como el sombreo y la doble fachada, para lograr una situación de confort en términos del modelo de confort adaptativo.

Como se demostró, los rangos exigidos por el concurso no corresponden a la situación climática tropical de Cali. Esta constatación lleva a la necesidad de revisar dichos rangos para unas próximas versiones de la competencia en entornos tropicales. Los rangos propuestos promueven una mayor exigencia térmica y, por tanto, la presencia de prototipos con acondicionamiento mecánico para alcanzar dichos valores, lo cual es poco común en la vivienda social en ciudades de América Latina. 
Por último, cabe mencionar que, para la competencia y los tiempos de ejecución, el prototipo no se pudo construir en CAR. Sin embargo, es un reto de implementación en el futuro. Por otro lado, es importante destacar que resultaron exitosas las medidas orientadas al aprovechamiento de residuos derivados del plástico de un solo uso, gracias a las cuales se generaron insumos para elementos de la envolvente, la siembra y otros componentes arquitectónicos, los que se explorarán a mayor profundidad una vez que el prototipo se convierta en Laboratorio de Vivienda, a cargo de las dos universidades involucradas en esta iniciativa.

\section{AGRADECIMIENTOS}

Se agradece a las Universidades de San Buenaventura, Cali y Autónoma de Occidente, como también al concurso Solar Decathlon, los cuales hicieron posible este proyecto. Se expresa especial reconocimiento a todo el equipo TUHOUSE conformado por estudiantes y docentes de las dos instituciones universitarias.

\section{REFERENCIAS BIBLIOGRÁFICAS}

ASHRAE/ANSI (2017). Standard 55-2017: Thermal Environmental Conditions for Human Occupancy. Pub. L. No. 55-2017, ASHRAE.

ASRHAE (2005). Thermal Comfort. En ASHRAE HandbookFundamentals.

Auliciems, A. (1975). Warmth and comfort in the subtropical winter: A study in Brisbane schools. Journal of Hygiene, 74(3), 339-343. DOI: https://doi.org/10.1017/S0022172400046854

Baumber, A., Kligyte, G., Bijl-Brouwer, M. Van Der y Pratt, S. (2020). Learning together: a transdisciplinary approach to student-staff partnerships in higher education. Higher Education Research \& Development, 39(3), 395-410.

Becker, R., Goldberger, I. y Paciuk, M. (2007). Improving energy performance of school buildings while ensuring indoor air quality ventilation. Building and Environment, 42(9), 3261-3276. DOI:10.1016/j.buildenv.2006.08.016

Bedoya, C. y Dzul, L. (2015). El concreto con agregados reciclados como proyecto de sostenibilidad urbana. Revista Ingenieria de Construccion, 30(2), 99-108. DOI: https://doi. org/10.4067/s0718-50732015000200002

Cobo, C., Villalobos, M. y Montoya, O. L. (2019). Sustainable architecture and engineering MIHOUSE project. Santiago de Cali: Editorial Bonaventuriana \& Universidad Autónoma de Occidente.

Evans, J. M. y Schiller, S. D. (1994). Diseño bioambiental y arquitectura solar. Buenos Aires: Universidad de Buenos Aires.

Fanger, P. O. (1972). Thermal comfort. Analysis and applications in environmental engineering. New York: McGraw-Hill.
Fanger, P. O. y Toftum, J. (2002). Extension of the PMV model to non-air-conditioned buildings in warm climates. Energy and Buildings, 34(6), 533-536. DOI: https://doi.org/10.1016/S03787788(02)00003-8

Gamboa, J. D., Rosillo, M. E., Herrera, C. A., López, O. y Iglesias, V. (2011). Confort Ambiental en vivienda de interés social en Cali. Santiago de Cali: Universidad del Valle.

Giraldo, W., Czajkowski, J. D. y Gómez, A. F. (2020). Confort térmico en vivienda social multifamiliar de clima cálido en Colombia. Revista de Arquitectura (Bogotá), 23(1).

Herrera, C. A. y Rosillo, M. (2019). Confort y eficiencia energética en el diseño de edificaciones: un enfoque práctico. Santiago de Cali: Universidad del Valle.

Herrera, R., Pineda, P., Roa, J., Cordero, S. y López, Á. (2017). Proyecto Aura: vivienda social sostenible. En 3er Congreso Internacional de Construcción Sostenible y Soluciones EcoEficientes (pp. 686-697). Recuperado de https://idus.us.es/ handle/11441/59216. Universidad de Sevilla, Escuela Técnica Superior de Arquitectura.

Herrera, R., Rey, J., Hernández, M. y Roa, J. (2020). Student competitions as a learning method with a sustainable focus in higher education: The University of Seville «Aura Projects» in the «Solar Decathlon 2019». Sustainability (Switzerland), 12(4). DOI: https://doi.org/10.3390/su12041634

Instituto Colombiano de Normas Técnicas y Certificación -ICONTEC (2004). Norma Técnica Colombiana NTC 5316. Thermal environmental conditions for human occupancy.

IRAM 11601 (2004). Aislamiento Térmico para edificios. Argentina. Recuperado de http://materias.fi.uba.ar/6731/Tablas/Tabla6.pdf

Konya, A. (1980). Design primer for hot climates. London: The Architectural Press Ltda.

López, Y. U. y Holguín, J. E. (Eds.). (2020). Water and Energy Engineering for Sustainable Buildings Mihouse Project. Santiago de Cali: Editorial Universidad Autónoma de Occidente.

Macías, H. (2020). Reporte final, grupo de energías equipo TUHOUSE. Santiago de Cali.

Montoya, O. L. (2014). Habitabilidad en los conjuntos multifamiliares de interés social construidos en Cali entre 1990 y 2010. Santiago de Cali: Editorial Bonaventuriana

Montoya, O. L. (2020). La arquitectura del aula para el trópico. Principios de diseño pasivo para edificaciones eficientes. Tesis de doctorado. Universidad Nacional de La Plata, Argentina.

Nicol, F., Humphreys, M. y Roaf, S. (2012). Adaptive thermal comfort: principles and practice. Londres: Routledge.

Olgyay, V. (1963). Clima y Arquitectura en Colombia. Barcelona: Gustavo Gili.

Samper, G. (2002). Recinto urbano: la humanización de la ciudad. Bogotá: Fondo Editorial Escala.

Samper, G. (2003). La evolución de la vivienda. Bogotá: Colección Somos Sur. 
San Juan, G., Rosenfeld, E., Santinelli, G., Discoll, C., Viegas, G., Brea, B., ... Rojas, D. (2013). Diseño bioclimático como aporte al proyecto arquitectónico. La Plata: Universidad de La Plata.

Sanchez, J. A. (2019). De la cubierta urbana al prototipo. Proceso evolutivo para el concurso Solar Decathlon Latinoamérica y el Caribe 2019. Sevilla. Recuperado de https://idus.us.es/ bitstream/handle/11441/89634/0\%20AO\%20Tfg\%20ETSA\%20 253.pdf?sequence $=1$

SOLAR DECATHLON (2019). Final rules. Solar Decathlon para América Latina y el Caribe. Colombia.

Terrados, F., Baco, L. y Moreno, D. (2015). Patio 2.12: Vivienda prefabricada, sostenible, autosuficiente y energéticamente eficiente. Participación en la competición Solar Decathlon Europe 2012. Informes de la Construcción, 67(538), 1-11.

UNIDAD DE PLANEACIÓN MINERO ENERGÉTICA -UPME (2018). Boletín estadístico de minas y energía. Recuperado de http://www1.upme.gov.co/PromocionSector/SeccionesInteres/ Documents/Boletines/Boletin_Estadistico_2018.pdf

UNIDAD DE PLANEACIÓN MINERO ENERGÉTICA-UPME (2019). Plan energético nacional 2020-2050. Recuperado de https:// www1.upme.gov.co/DemandaEnergetica/PEN_documento_ para_consulta.pdf\#search $=$ consumo energia sector residencial

Van der Vegt, A. y Govaert, L. E. (2005). Polymeren, van keten tot kunststof. Amsterdam: VSSD.

Zapata, C. M., Viegas, G. M., San Juan, G. A., Ramos, H., Coronado, J. A., Ochoa, J., ..., y Montoya, O. L. (2018). Comodidad ambiental en aulas escolares. Incidencia en la salud docente y en el rendimiento cognitivo de los estudiantes en colegios públicos de Bogotá, Medellín y Cali. Santiago de Cali: Editorial Bonaventuriana, Ediciones Unisalle, Universidad Nacional de la Plata. Recuperado de http://www.editorialbonaventuriana.usb. edu.co/libros/2018/comodidad-ambiental-aulas/index.html 